\title{
Assistance au suicide - un témoignage fort sur des enjeux complexes
}

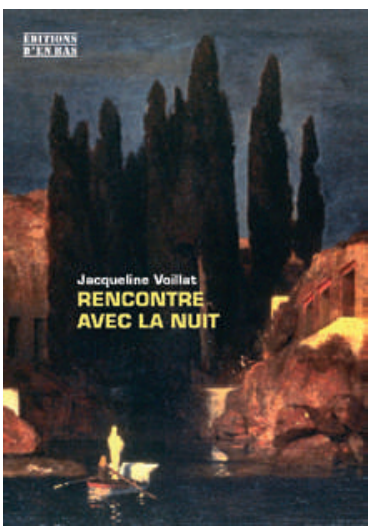

Couverture du livre de Jacqueline Voillat [2].
Le canton de Zurich a connu en mai 2011 deux votations sur l'assistance au suicide et d'éventuels aménagements en rapport avec l'article 115 du Code pénal suisse, libéral, ouvert (sauf motifs égoïstes, l'aide apportée à un suicidant n'est pas punissable). Les citoyens vaudois eux ont accepté le 17 juin dernier un article $27 \mathrm{~d}$ de la loi sur la santé publique commençant par «Les établissements sanitaires reconnus d'intérêt public ne peuvent refuser la tenue d'une assistance au suicide en leur sein, demandée par un patient, si les conditions suivantes sont remplies...» (la décision elle-même étant celle du patient capable de discernement gravement et incurablement malade).

La volonté de notre population de conserver pour chacun cette liberté de disposer de lui-même apparaît claire. Si le Conseil fédéral a jugé à deux reprises que le statu quo (art. 115 CPS sans autre ajout de niveau légal) pourrait bien être le moins mauvais système, le débat reste ouvert au plan sociétal et de l'éthique: sociale, professionnelle et éthique de chacun. J'estime très important que jamais les pouvoirs publics ne donnent l'impression qu'ils cautionnent le suicide, qui serait vu comme une option comme une autre. C'est le risque de dispositions supplémentaires de droit public (cela peut valoir pour la nouvelle norme vaudoise) qu'elles paraissent le légitimer. L'expérience m'a convaincu qu'il y a des choses que l'Etat et la loi ne savent pas faire adéquatement, en particulier s'agissant de questions fondamentalement privées - telles que le suicide. En cette matière, il ne peut y avoir d'attitude «optimale» valable pour chacun, ni de règle générale. Ainsi que la Commission nationale d'éthique l'a relevé [1], chaque situation doit être considérée pour elle-même, dans ses différentes dimensions, avec la personne concernée, en dialogue avec les proches signifiants (y compris soignants) et le médecin auquel est demandée la prescription létale.

Les histoires individuelles sont toutes différentes... Un témoignage vient d'être publié qui mérite de retenir l'attention [2]: description d'un noyau familial (une mère très âgée et ses deux filles) aux prises avec la demande réitérée, parfois agressive, de la mère d'avoir recours à Exit. Avec des flashbacks sur les décennies antérieures, le récit porte surtout sur la période de six mois que la vieille dame passe en hôpital gériatrique après avoir dû quitter son domicile (elle ne peut plus se déplacer qu'en chaise roulante).
L'auteure, une des filles, décrit par le détail les rapports de sa sœur et elle avec leur mère, marqués par beaucoup de paroles vives (jusqu'à la haine - sic), sur le fond d'une vie familiale qui a eu ses difficultés mais à laquelle les sœurs restent attachées par de nombreux bons souvenirs. Description aussi des contacts et discussions avec les médecins et équipes soignantes qui illustre comment, si bien sûr nous vivons dans un monde imparfait et si certains professionnels sont moins bons que d'autres dans leurs relations, il reste en général que chacun s'efforce de remplir son rôle correctement, en fonction des règles de la profession. Néanmoins, les moments de tension voire de colère sont nombreux, au milieu des hésitations et questionnements multiples, que ce soit sur des points philosophiques ou pratiques.

Finalement, il est fait appel à Exit, un médecin de cette association voit la patiente et ses filles à plusieurs reprises, ainsi que le médecin hospitalier, et on s'achemine vers l'aide au suicide, fixée trois semaines à l'avance. Après les turbulences et ambivalences autour de la demande de la vieille dame, le récit des sentiments des protagonistes durant cette dernière période est fort, prenant - spécialement celui des jours précédant et suivant immédiatement le décès. A noter le soutien substantiel du pasteur de leur localité de domicile, qui les voit et est présent lors du suicide. Dans la postface, J. Voillat relève qu'il lui a fallu trois ans pour honorer la promesse faite à sa mère d'écrire son histoire et elle rend hommage aux professionnels de santé, confrontés à une situation inhabituelle et à la volonté opiniâtre de la patiente. «'ai admiré qu'ils arrivent à dépasser leurs principes, leurs doutes, à accepter ce que ma mère voulait si fort, à l'entourer jusqu'à la fin de soins, de respect et peut-être d'affection.»

L'auteure, enseignante, a un talent certain d'écriture. C'est un témoignage qui éclaire la problématique de l'assistance au suicide et les enjeux qui y sont liés, pour la personne concernée et ses proches. Dans les termes d'une lectrice avisée: un livre «sur les doutes, les déchirements et finalement les certitudes dans ce qui apparaît comme un combat à couper le souffle (...) un récit qui, loin du plaidoyer, s'ouvre à toutes les lectures» [3].

Jean Martin, ancien médecin cantonal, membre de la rédaction et de la Commission nationale d'éthique 